\title{
What's in a name? Hilo-Jejunostomy, not Hepatico-Jejunostomy, for Post Cholecystectomy Iatrogenic Benign Biliary Stricture
}

\author{
Vinay K. Kapoor* \\ Professor of Surgical Gastroenterology, Sanjay Gandhi Post-graduate Institute of Medical Sciences (SGPGIMS), Lucknow 226014 UP, India
}

Received: 08 April, 2016; Accepted: 29 June, 2016; Published: 10 July, 2016

*Corresponding author: Professor of Surgical Gastroenterology, Sanjay Gandhi Post-graduate Institute of Medical Sciences (SGPGIMS), Lucknow 226014 UP, India, E-mail: vkkapoor.india@gmail.com

\section{Background}

Gall stone disease is common all over the world; cholecystectomy is one of the commonest operations performed by a general surgeon. Bile duct injury is a not uncommon complication of cholecystectomy and the risk of bile duct injury is at least 2-3 times more during laparoscopic than during open cholecystectomy, the incidence being around $0.5 \%$.

Bile duct injury during cholecystectomy may result in an iatrogenic benign biliary stricture. Benign biliary strictures are classified from Bismuth Type I to Type $\mathrm{V}$ depending upon the length of the common hepatic duct stump and patency of the biliary ductal confluence as follows

Type I Common hepatic duct stump $>2 \mathrm{~cm}$

Type II Common hepatic duct stump $<2 \mathrm{~cm}$

Type III No common hepatic duct stump but biliary ductal confluence patent

Type IV Biliary ductal confluence not patent (right and left hepatic ducts separated)

Type V Stricture involving an aberrant duct

The gold standard of management of benign biliary stricture is surgical repair in the form of a biliary enteric anastomosis. Biliary enteric anastomosis for benign biliary stricture, irrespective of its Bismuth Type, should be at the hilum of the liver. The hilar plate needs to be lowered in order to expose the extra-hepatic horizontal part of the left hepatic duct at the base of the quadrate lobe (segment IV). The ductotomy should extend across the hilum (biliary ductal confluence) into the left hepatic duct- the Hepp Couinaud approach [1].

We want to suggest a new name for the biliary-enteric anastomosis performed for benign biliary stricture.

\section{Surgical anatomy}

Right and left hepatic ducts unite at the hilum of the liver to form the common hepatic duct which is joined by the cystic duct and continues as the common bile duct. Extrahepatic bile ducts, common hepatic duct and common bile duct, receive their blood supply from below from the gastroduodenal artery or the proper hepatic artery [2] via two longitudinal arteries which run along the length of the bile duct at 3 and 9' 0 clock positions and from above from the caudate artery and the medial subsegmental artery of segment IV via an arterial network present in the hilum of the liver [3]. In the case of a bile duct injury, the blood supply from below has been interrupted and the only blood supply to the bile ducts is from the hilum. For this reason, even in a Bismuth Type I or II BBS, the common hepatic duct stump though present has poor blood supply and is not suitable for anastomosis.

\section{Discussion}

The biliary-enteric anastomosis for benign biliary stricture is thus different from other biliary-enteric anastomosis as follows:

i. Choledocho-duodenostomy (Figure 1) for stone disease where the infra-cystic supra-duodenal part of the common bile duct is anastomosed to the first part of the duodenum.

ii. Hepatico-jejunostomy (Figure 2) in pancreatoduodenectomy or after excision of a choledochal cyst where the common hepatic duct above the cystic duct but below the hilum is anastomosed to a Roux-en-Y loop of jejunum.

iii. Porto-enterostomy (Figure 3) for extra-hepatic biliary atresia where the liver capsule around the porta hepatis containing openings of multiple intrahepatic bile ducts is anastomosed to a Roux-en-Y loop of jejunum.

iv. Cholangio-jejunostomy (Figure 4) for high biliary block e.g. hilar cholangiocarcinoma [4] or gall bladder cancer [5] where an intra-hepatic bile duct, most commonly of segment III, is anastomosed to a Roux-en-Y loop of jejunum.

v. Longmire procedure, again for the high biliary block where a part of the left lateral segment of the liver is resected 


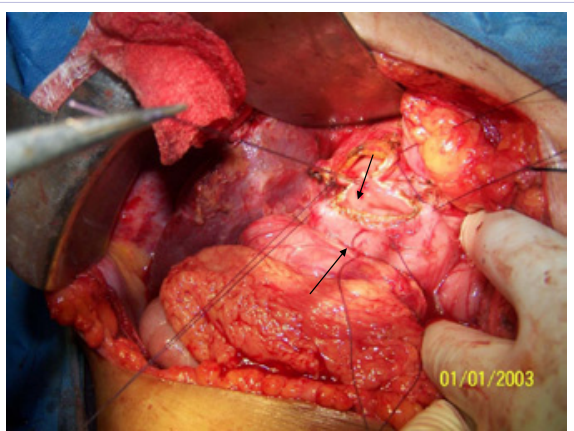

Figure 1: Choledocho-duodenostomy-side to side anastomosis between the infra-cystic supra-duodenal part of the common bile duct (short arrow) and the first part of the duodenum (long arrow).

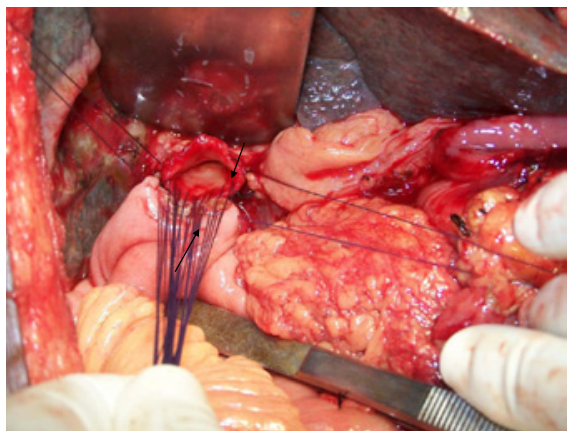

Figure 2: Hepatico-jejunostomy-end to side anastomosis between the common hepatic duct (short arrow) above the cystic duct but below the hilum and a Roux-en-Y loop of jejunum (long arrow).

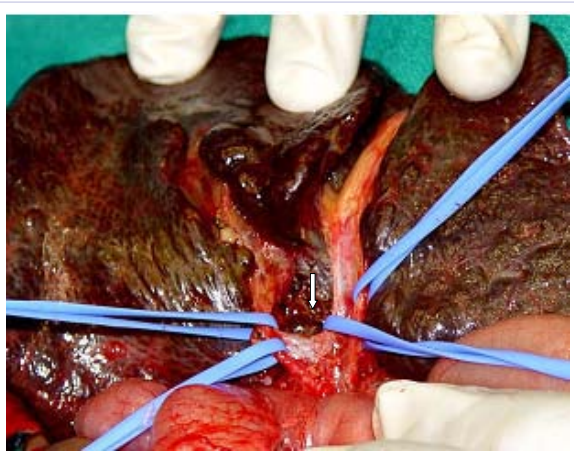

Figure 3: Porto-enterostomy -porta hepatis has been dissected and vessels have been looped; no bile ducts are seen-the liver capsule around the porta hepatis (short arrow) containing unseen openings of multiple small intra-hepatic bile ducts will be anastomosed to a Roux-en-Y loop of jejunum. Picture courtesy Prof Richa Lal Pediatric Surgery SGPGIMS Lucknow

so that the intrahepatic ducts of segments II and III are exposed; the cut surface of the liver is then anastomosed to a Roux-en-Y loop of jejunum. In a modification, the exposed intrahepatic ducts are anastomosed to the Rouxen-Y loop of jejunum [6].

\section{Proposal}

Cholangiocarcinoma arising from the bile ducts at the hilum (confluence of right and left hepatic ducts) is called hilar cholangiocarcinoma. Following the same nomenclature, we propose that the biliary-enteric anastomosis for postcholecystectomy benign biliary stricture which should be performed at the hilum (confluence of right and left hepatic ducts and extending into the left hepatic duct) should be called 'hilojejunostomy' (Figure 5) to differentiate it from other biliaryenteric anastomoses including hepaticojejunostomy.

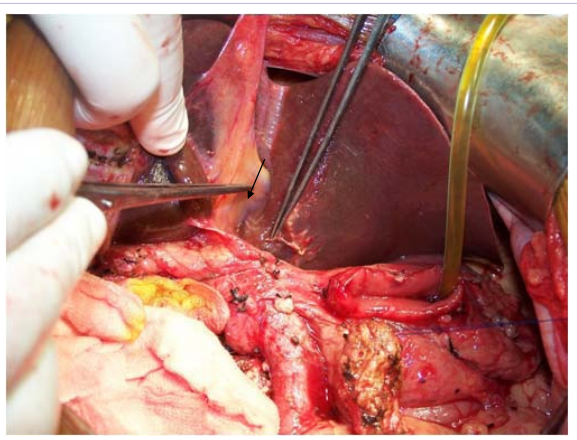

Figure 4: Cholangio-jejunostomy -the left forceps points to the left side of the base of the falciform (round) ligament where the intra-hepatic bile duct of segment III (short arrow) will be found and will be anastomosed to a Roux-en-Y loop of jejunum.

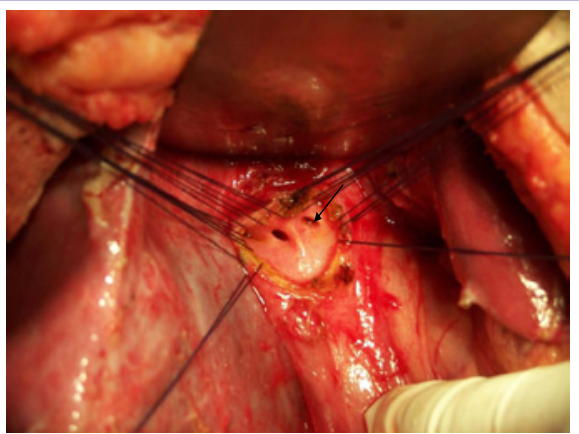

Figure 5: Hilo-jejunostomy-side to side biliary enteric anastomosis at the hilum (confluence of right and left hepatic ducts and extending into the left hepatic duct)-openings of both right and left hepatic ducts are seen (short arrow).

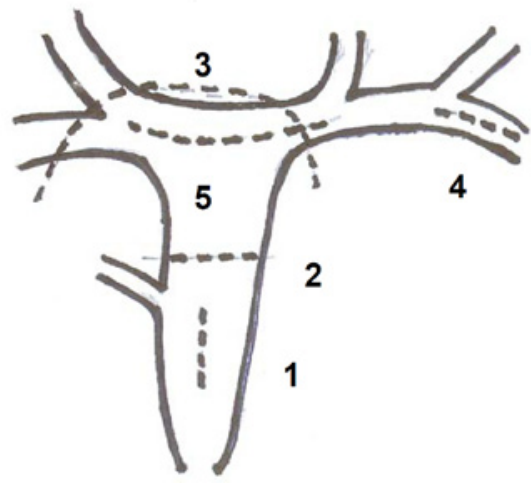

Figure 6: Diagrammatic representation of the sites of anastomosis: 1 Choledocho-duodenostomy, 2 Hepatico-jejunostomy, 3 Porto-enterostomy, 4 Cholangio-jejunostomy, 5 Hilo-jejunostomy. 


\section{References}

1. Hepp J, Couinaud C. [Approach to and use of the left hepatic duct in reparation of the common bile duct]. Presse Med. 1956;64(41):947948.

2. Dai J, Wu XF, Yang C, Li HJ, Chen YL, Liu GZ, et al. Study of relationship between the blood supply of the extrahepatic bile duct and duct supply branches from gastroduodenal artery on imaging and anatomy. Chin Med J (Engl). 2015;128(3):322-326. doi: 10.4103/0366-6999.150097.

3. Miyayama S, Yamashiro M, Hashimoto M, Hashimoto N, Ikuno M, Okumura K, et al. Blood supply of the main bile duct from the caudate artery and medial subsegmental artery of the hepatic artery:
Evaluation using images obtained during transcatheter arterial chemoembolization for hepatocellular carcinoma. Hepatol Res. 2013;43(11):1175-1181. doi: 10.1111/hepr.12071.

4. Connor S, Wigmore SJ, Madhavan KK, Parks RW, Garden OJ. Surgical palliation for unresectable hilar cholangiocarcinoma. HPB (Oxford). 2005;7(4):273-277. doi: 10.1080/13651820500372442.

5. Kapoor VK, Pradeep R, Haribhakti SP, Singh V, Sikora SS, Saxena $\mathrm{R}$, et al. Intrahepatic segment III cholangiojejunostomy in advanced carcinoma of the gallbladder. Br J Surg. 1996;83(12):1709-1711.

6. Traverso LW. The Longmire I, II, and III operations. Am J Surg. 2003;185(5):399-406. 